\title{
Prenatal exposure to zinc oxide particles alters monoaminergic neurotransmitter levels in the brain of mouse offspring
}

\author{
Yuka Okada ${ }^{1,{ }^{*},}$ Ken Tachibana ${ }^{1,3,{ }^{*},}$ Shinya Yanagita ${ }^{2,3,4}$ and Ken Takeda ${ }^{1,2,3}$ \\ 'Department of Hygienic Chemistry, Faculty of Pharmaceutical Sciences, \\ Tokyo University of Science, 2641 Yamazaki, Noda, Chiba 278-8510, Japan \\ ${ }^{2}$ Research Center for Health Science of Nanoparticles, Research Institute for Science and Technology, \\ Tokyo University of Science, 2641 Yamazaki, Noda, Chiba 278-8510, Japan \\ ${ }^{3}$ The Center for Environmental Health Science for the Next Generation, Research Institute for Science and \\ Technology, Tokyo University of Science, 2641 Yamazaki, Noda, Chiba 278-8510, Japan \\ ${ }^{4}$ Faculty of Science and Technology, Tokyo University of Science, 2641 Yamazaki, Noda, Chiba 278-8510, Japan
}

(Received February 8, 2013; Accepted March 6, 2013)

\begin{abstract}
Zinc oxide ( $\mathrm{ZnO}$ ) nano-sized particles (NPs) are beneficial materials used for sunscreens and cosmetics. Although ZnO NPs are widely used for cosmetics, the health effects of exposure during pregnancy on offspring are largely unknown. Here we investigated the effects of prenatal exposure to $\mathrm{ZnO}$ NPs on the monoaminergic system of the mouse brain. Subcutaneous administration of ZnO NPs to the pregnant ICR mice (total $500 \mu \mathrm{g} /$ mouse) were carried out and then measured the levels of dopamine (DA), serotonin (5-HT), and noradrenalin, and their metabolites in 9 regions of the brain of offspring (6-week-old) using high performance liquid chromatography (HPLC). HPLC analysis demonstrated that DA levels were increased in hippocampus in the ZnO NP exposure group. In the levels of DA metabolites, homovanillic acid was increased in the prefrontal cortex and hippocampus, and 3, 4-dihydroxyphenylacetic acid was increased in the prefrontal cortex by prenatal $\mathrm{ZnO}$ NP exposure. Furthermore, DA turnover levels were increased in the prefrontal cortex, neostriatum, nucleus accumbens, and amygdala in the $\mathrm{ZnO} \mathrm{NP}$ exposure group. We also found changes of the levels of serotonin in the hypothalamus, and of the levels of 5-HIAA (5-HT metabolite) in the prefrontal cortex and hippocampus in the ZnO NPexposed group. The levels of 5-HT turnover were increased in each of the regions except for the cerebellum by prenatal ZnO NP exposure. The present study indicated that prenatal exposure to ZnO NPs might disrupt the monoaminergic system, and suggested the possibility of detrimental effects on the mental health of offspring.
\end{abstract}

Key words: Brain, Monoaminergic neurotransmitter, Nanoparticle, Prenatal exposure, Zinc oxide

\section{INTRODUCTION}

Nano-sized particles (NPs) are widely used for medicine, industrial products, and cosmetics (Morabito et al., 2011; Kuan et al., 2012). In particular, NPs of zinc oxide $(\mathrm{ZnO})$ and titanium dioxide $\left(\mathrm{TiO}_{2}\right)$ are beneficial to sunscreens and foundation because these particles are colorless and reflect ultraviolet rays (Nohynek et al., 2007). While the physical and chemical characteristics of NPs make them useful for many industrial applications, previous studies revealed that NPs can enter the systemic circulation, and migrate to various organs such as the liver, kidney, spleen, and heart (Kreyling et al., 2009; Liu et al., 2009; Oberdörster et al., 2009). Some NPs induce production of inflammatory cytokines (Oberdörster, 2001; Beckett et al., 2005; Tin Tin Win et al., 2006), and affect the monoaminergic neurotransmitters (Tin Tin Win et al., 2008). Monoaminergic neurotransmitters

Correspondence: Ken Takeda (E-mail: takedak@rs.noda.tus.ac.jp)

Ken Tachibana (E-mail: ktachiba@rs.noda.tus.ac.jp)

*These authors equally contributed to this work. 


\section{Y. Okada et al.}

such as dopamine (DA), noradrenaline (NA), and serotonin (5-HT) play a pivotal role in several physiological and psychological functions. Several reports have suggested that monoaminergic system disruption is related to psychiatric disorders including schizophrenia, depression, autism, and attention-deficit hyperactivity disorder (Froehlich et al., 2010; Nakamura et al., 2010; Paclt et al., 2009).

We previously demonstrated that nano-sized $\mathrm{TiO}_{2}$, administered to pregnant mice, was transferred to the offspring and affected the reproductive and central nervous system of male offspring (Takeda et al., 2009). Because a fetus is highly vulnerable to environmental stimuli, chemical exposure in the fetal period generally disrupts its development (Kawashiro et al., 2008; Needham and Sexton, 2000). Although these aspects suggest that the detrimental health effects of the NPs would be more profound in fetuses than in adults, the potential toxicity of prenatal exposure of NPs is less investigated. We recently reported that prenatal exposure to $\mathrm{TiO}_{2}$ NPs affects gene expression related to the development and function of the central nervous systems including the monoaminergic system (Shimizu, et al. 2009). Because ZnO NPs are also widely used in cosmetics, there is a concern with the health effects of prenatal $\mathrm{ZnO}$ NP exposure on offspring. Previous studies suggest that ZnO NPs induce cytotoxicity and oxidative stress in primary mouse embryonic fibroblast (Yang et al., 2009) and developmental disorder in Zebrafish (Zhu et al., 2008); however its health effects on offspring exposed to $\mathrm{ZnO}$ NPs during the fetal period are largely unknown. In the present study, we investigated the effects of prenatal exposure to $\mathrm{ZnO}$ NPs on the monoaminergic systems. We comprehensively examined the levels of monoamines and their metabolites in 9 regions of the brain in mice using high performance liquid chromatography (HPLC).

\section{MATERIALS AND METHODS}

\section{ZnO particles}

Mz-300 NPs, ZnO with a primary diameter of 30-40 nm, were kindly provided by Tayca Co. (Osaka, Japan). ZnO NPs were dispersed in saline containing $0.05 \%$ Tween- 80 , and the sample suspension was sonicated for $90 \mathrm{~min}$ in a bath-type sonicator immediately before administration. To minimize the heat production, the bath was filled with enough volume of water. A wide distribution of $\mathrm{ZnO}$ powder of different diameters was confirmed by field emission-type scanning electron microscopy. The size distribution of the ZnO NPs in the suspension was measured by dynamic light scattering using an FPAR-1000
(Otsuka Electronics Co., Ltd., Osaka, Japan). Size distribution of ZnO NPs was assessed with the CONTIN algorithm to obtain the diameter distribution of polydispersed particles from dynamic light scattering data.

\section{Animals}

Pregnant ICR mice (8-11-week-old) at gestation day (GD) 1 were purchased from SLC Co. (Shizuoka, Japan). $\mathrm{ZnO}$ NPs were suspended at $0.5 \mathrm{mg} / \mathrm{ml}$, and $0.2 \mathrm{ml}$ was administrated subcutaneously to the pregnant ICR mice at GD 5, 8, 11, 14, and $17(100 \mu \mathrm{g} /$ mouse/day: total $500 \mu \mathrm{g} / \mathrm{mouse})$. Administered $\mathrm{ZnO}$ NPs was same as the mass dose in the previous study about the effects of prenatal $\mathrm{TiO}_{2} \mathrm{NP}$ exposure on dopaminergic system (Takahashi et al., 2010). Control mice were treated with saline containing $0.05 \%$ Tween- 80 . In each group, pups were weaned on postnatal day 21 . They were housed under controlled conditions with a $12 \mathrm{hr}$ light/ $12 \mathrm{hr}$ dark cycle and ad libitum access to food and water. All experiments were handled in accordance with the institutional and national guidelines for the care and use of laboratory animals. All efforts were made to minimize the number of animals used and their suffering.

\section{HPLC analysis of neurotransmitters}

Brains were removed from 6-week-old anesthetized male pups ( $\mathrm{n}=8 /$ group). Only male mice were analyzed in this study because the neurotransmitter variations were seen during different stages of the estrous cycle in female mice (Dazzi et al., 2007; Xiao and Becker, 1994). Serial coronal sections of the brain (2 mm thick) were obtained using a Rodent brain slicer (MUROMACHI KIKAI, Tokyo, Japan) and dissected to 9 regions: prefrontal cortex, neostriatum (caudate-putamen), nucleus accumbens, hippocampus, amygdala, hypothalamus, midbrain, brainstem, and cerebellum. The dissected regions were immediately frozen in liquid nitrogen and stored at $-80^{\circ} \mathrm{C}$ until use.

Frozen brain tissue was homogenized in ice-cold perchloric acid containing $100 \mathrm{mM}$ EDTA $(2 \mathrm{Na})$ and $100 \mathrm{ng}$ isoproterenol as an internal standard. The homogenates were centrifuged at $20,000 \times \mathrm{g}$ for $15 \mathrm{~min}$ at $0^{\circ} \mathrm{C}$. Supernatants were transferred to new tubes and the pellets were stored for protein assay. The $\mathrm{pH}$ of the supernatant was adjusted to 3.0 with $1 \mathrm{M}$ sodium acetate, and stored at $-80^{\circ} \mathrm{C}$ until use. For HPLC analysis, $10 \mu \mathrm{l}$ of the $\mathrm{pH}$-adjusted supernatant were injected into an HPLC system with electrochemical detection (EICOM Co., Kyoto, Japan). Monoamines were separated by a C18 reverse-phase column (EICOMPAK SC-5ODS, EICOM) with a mobile phase containing sodium acetate and citric 
acid. The mobile phase was prepared as follows: $0.1 \mathrm{M}$ sodium acetate was mixed with $0.1 \mathrm{M}$ citric acid in a 10:9 ratio, and was adjusted to $\mathrm{pH} 3.5$ (0.1 M sodium acetatecitric acid buffer), mixed with methanol in a ratio of $85: 15$ and then supplemented with sodium 1-octanesulfonate (100 mg/l), EDTA (2Na) (5 mg/ml). DA, 3, 4-dihydroxyphenylacetic acid (DOPAC), homovanillic acid (HVA), 3-methoxytyramine (3-MT), NA, normetanephrin (NM), 3-methoxy-4-hydrophenyl (MHPG), 5-HT, and 5-hydroxyindole-3-acetic acid (5-HIAA) were analyzed.

\section{Protein assay}

Pellets were resuspended in $100 \mathrm{mM}$ Tris- $\mathrm{HCl}(\mathrm{pH}$ 10.4) and protein concentration was determined using Advanced Protein Assay Reagent (Cytoskeleton Inc., Denver, CO, USA). Measurements were performed according to the manufacturer's protocol.

\section{Statistical analysis}

The data were expressed as mean \pm S.E.M. Differences between groups were examined for statistical significance using a Mann-Whitney $U$-test. $P<0.05$ indicated statistical significance.

\section{RESULTS}

\section{Size distribution and agglomeration state in suspension of ZnO NPs}

A scanning electron microscope image of $\mathrm{ZnO}$ NPs dispersed in saline containing $0.05 \%$ Tween- 80 is shown in Fig. 1A. ZnO NPs were slightly aggregated. The size distribution of secondary ZnO NPs in the suspension, analyzed by dynamic light scattering, ranged from 80 to $700 \mathrm{~nm}$ with $190 \mathrm{~nm}$ being the mode value (Fig. 1B).

\section{Monoamine levels in 9 regions of the brain in ZnO NP-exposed mice}

We first examined the effect of prenatal exposure to $\mathrm{ZnO}$ NPs on the levels of DA and its metabolites (DOPAC, HVA, and 3-MT; Fig. 2, Supplementary Table 1). DOPAC and HVA were significantly increased in the prefrontal cortex of $\mathrm{ZnO}$ NP-exposed mice (DOPAC, + 49.1\%; HVA, +28.4\%; Fig. 2). DA and HVA were significantly increased in the hippocampus. Moreover, DA levels were decreased in the cerebellum. 3-MT level was not altered significantly in any of the regions of the brain examined in the present study. Next, we calculated the metabolic turnover of DA ((DOPAC $+\mathrm{HVA}+3 \mathrm{MT}) / \mathrm{DA})$, and found that it was significantly increased in the prefrontal cortex, neostriatum, nucleus accumbens, and amygdala in the $\mathrm{ZnO}$ NP-exposed group.

5-HT level was decreased in the hypothalamus, and 5-HIAA level was increased in the prefrontal cortex and hippocampus in $\mathrm{ZnO}$ NP-exposed mice (Fig. 3, Supplementary Table 2). The metabolic turnover of 5-HT (5-HIAA/5-HT) was increased in 8 regions of $\mathrm{ZnO} \mathrm{NP}-$ exposed mice (Fig. 3, Supplementary Table 2).

We then measured the levels of NA and its metabolites (NM, MHPG; Supplementary Fig. 1, Supplementary Table 3). NM level was increased in the hippocampus
(A)

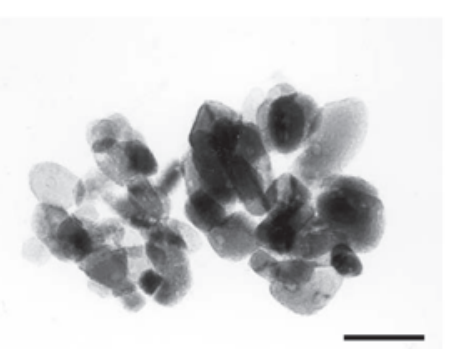

(B)

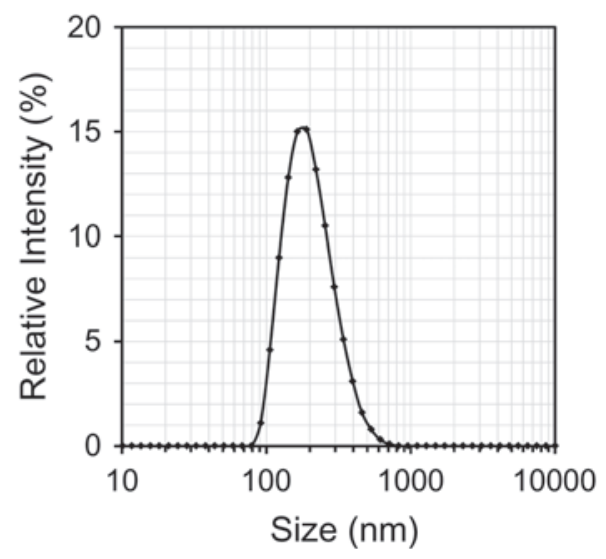

Fig. 1. Field-emission-type scanning electron microscopy images of ZnO NPs and their size distribution. ZnO NPs were suspended in saline with $0.05 \%(\mathrm{v} / \mathrm{v})$ Tween-80 and were sonicated for $90 \mathrm{~min}$ immediately before administration. (A) The agglomeration state was assessed by field emission-type scanning electron microscopy (Scale bar $=50 \mathrm{~nm}$ ). (B) The size distribution of $\mathrm{ZnO}$ NPs in the suspension was analyzed by dynamic light scattering. 
Y. Okada et al.
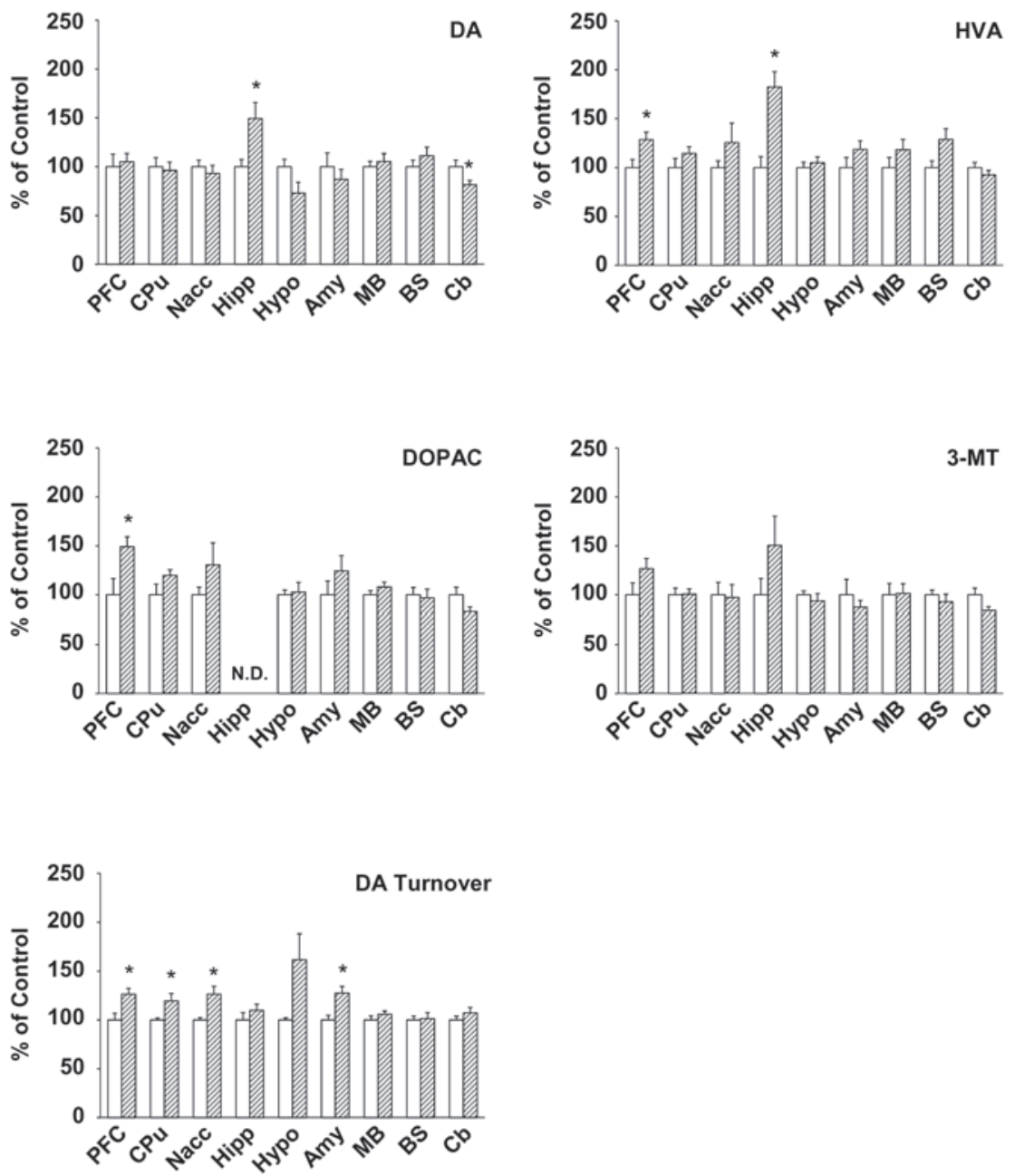

Fig. 2. Effects of prenatal $\mathrm{ZnO} \mathrm{NP}$ exposure on DA and their metabolites level in the brain. DA turnover was calculated as (DOPAC+HVA+3-MT)/DA. Each column (Control group, open column; ZnO NP group, forward slash column) represents the mean \pm S.E.M. ${ }^{*} \mathrm{p}<0.05$ vs each control group. Abbreviations: PFC, Prefrontal cortex; $\mathrm{CPu}$, Neostriatum (CaudatePutamen); Nacc, Nucleus accumbens; Hipp, Hippocampus; Hypo, Hypothalamus; Amy, Amygdala; MB, Midbrain; BS, Brainstem; $\mathrm{Cb}$, Cerebellum. N.D.: Not detectable.

of $\mathrm{ZnO}$ NP-exposed mice. In addition, MHPG level was decreased in the hypothalamus, and cerebellum. NA level was not altered significantly in any of the regions of the brain examined in the present study. The metabolic turnover of the NA was decreased in the hypothalamus, and amygdala of the $\mathrm{ZnO} \mathrm{NP}$-exposed mice.

\section{DISCUSSION}

Developmental disorders of the brain caused by genetic and environmental factors are of great significance to psychiatric health. Previous studies indicated an associa- tion between chemical exposure in the environment and monoaminergic neurotransmitter regulation (Matsuda et al., 2010; Xia et al., 2011; Sirivelu et al., 2006). The monoaminergic system plays an important role in mental health, and its dysregulation leads to psychiatric dysfunction, such as behavioral depression, anxiety disorder, and Parkinsonism (Weiss et al., 1981; Elsworth and Roth, 1997; Morilak and Frazer, 2004). It is noteworthy that prenatal stress can cause profound and long-lasting deficits in brain functions (Chung et al., 2005; Son et al., 2006). Maternally-stressed male mice have been shown to indicate altered dopaminergic responses (Son 
Prenatal $\mathrm{ZnO}$ nanoparticle exposure alters monoamines in mouse brain
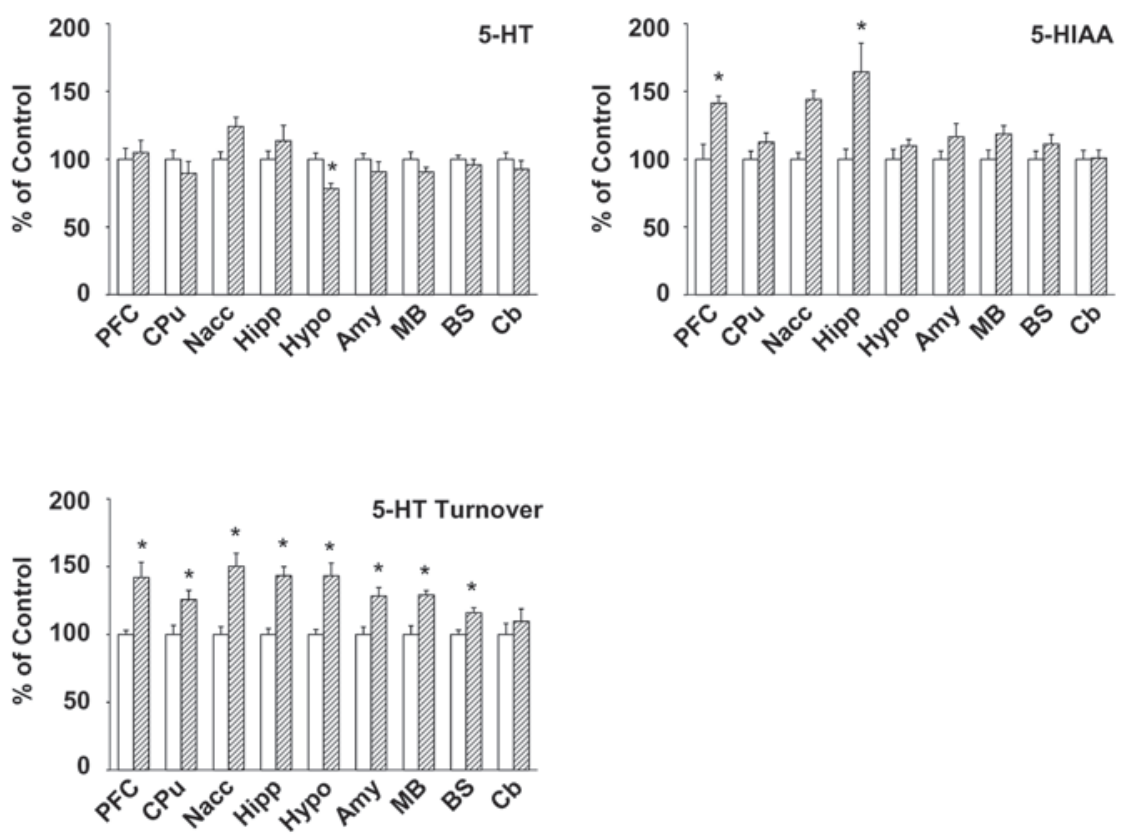

Fig. 3. Effects of prenatal $\mathrm{ZnO} \mathrm{NP}$ exposure on 5-HT and their metabolites level in the brain. The 5-HT turnover was calculated as 5-HIAA/5-HT. Each column (Control group, open column; ZnO NP group, forward slash column) represents the mean \pm S.E.M. ${ }^{*} p<0.05$ vs each control group. Abbreviations: PFC, Prefrontal cortex; CPu, Neostriatum (Caudate-Putamen); Nacc, Nucleus accumbens; Hipp, Hippocampus; Hypo, Hypothalamus; Amy, Amygdala; MB, Midbrain; BS, Brainstem; $\mathrm{Cb}$, Cerebellum.

et al., 2007). Furthermore, Narita et al. (2002), showed that fetal thalidomide or valproic acid exposure resulted in increased monoamine concentration in the brain, and induced an autism-like phenotype in rats.

In the present study, we comprehensively analyzed the amount of monoamines and their metabolites using HPLC in adult offspring mice. In the $\mathrm{ZnO}$ NP-exposed group, metabolic turnover of DA and 5-HT was significantly increased in the prefrontal cortex, neostriatum, nucleus accumbens, and amygdala. In the prefrontal cortex in particular, the levels of DA metabolites (DOPAC and HVA) and a 5-HT metabolite (5-HIAA) were significantly increased. 5-HT neurons of the raphe nucleus are projected to prefrontal cortex and neostriatum, and the activity of 5-HT and DA neuron systems are mutually regulated (Assié et al., 2005). Oades (2008) reported that the metabolic turnover of DA and 5-HT are simultaneously increased in attention-deficit hyperactivity disorder patients. These reports reveal the importance of the balance of activity and metabolism of DA and 5-HT in the monoaminergic system of the brain. Combined with these reports, prenatal exposure to $\mathrm{ZnO}$ NPs induced monoaminergic neurotransmitter disruption, in particular in the imbalance of DA and 5-HT levels, which potentially affects the mental and behavioral health of offspring. On the other hand, increased serotonin turnover in the prefrontal cortex and increased dopamine turnover in hippocampus, which were also observed in this study, were associated with interferon- $\alpha$-induced depression (De La Garza and Asnis, 2003). Moreover, Barton et al. (2008) reported that brain serotonin turnover was elevated in patients with depression. These reports suggest the prenatally $\mathrm{ZnO}$-exposed mice represent depression-like behavior. Further behavioral tests should be performed to solve the effects of prenatal $\mathrm{ZnO}$ NP exposure on the mental and behavioral health of offspring.

Synthesized neurotransmitters are stored in synaptic vesicles and then released from vesicles when action potential reaches the synaptic terminal. Released neurotransmitters are immediately inactivated by reuptake or metabolism. Although we measured the activity of monoamine oxygenase (a key enzyme in the metabolism of DA, NA, and 5-HT) in the prefrontal cortex and nucleus accumbens, we did not detect any significant alteration (Supplementary Fig 2). Because the increase of metabolic turnover depends on the increase of the amount of 


\section{Y. Okada et al.}

monoamines present in synaptic cleft, further examination regarding this point may reveal the mechanism of $\mathrm{ZnO}$ NP-mediated increase of metabolic turnover of monoamines.

Although the mechanism of monoaminergic system disruption due to prenatal $\mathrm{ZnO} \mathrm{NP}$ exposure is unclear, one possible mechanism is that prenatal exposure to $\mathrm{ZnO}$ NPs induced reactive oxygen species (ROS), which may influence the function of the monoaminergic system in offspring. Previous studies have reported that exposure to NPs, including $\mathrm{ZnO}$ NPs, produces ROS, and induces oxidative stress (Long et al., 2006; Xia et al., 2008). ROS-induced oxidative stress would cause functional impairment of the monoaminergic system via dysregulation of monoaminergic neurotransmitter metabolism. Monoaminergic systems may also be disrupted by $\mathrm{Zn}^{2+}$,which dissolves from $\mathrm{ZnO}$ NPs. Cho et al. (2011) showed that the toxicity of dissolved $\mathrm{Zn}^{2+}$ from $\mathrm{ZnO}$ NPs is much greater than a similar mass of ZnO NPs. Previous reports indicated that $\mathrm{Zn}^{2+}$ suppresses the function of the $\mathrm{GABA}_{\mathrm{A}}$ receptor through allosteric mechanism (Hosie et al., 2003). Taken together with the report which showed $\mathrm{GABA}_{\mathrm{A}}$ receptors regulate dopamine release in the prefrontal cortex (Santiago et al., 1993), an increase in $\mathrm{Zn}^{2+}$ caused by prenatal $\mathrm{ZnO}$ NP exposure may affect the release and metabolism of DA through suppression of the $\mathrm{GABA}_{\mathrm{A}}$ receptor function. Additionally, the monoamine transporters may be disrupted by dissolved $\mathrm{Zn}^{2+}$ from $\mathrm{ZnO}$ NPs. Previous report indicated that $\mathrm{Zn}^{2+}$ plays a role in the monoamine transporters associated with monoamine metabolism (Scholze et al., 2002). Further investigations of the interaction of prenatal $\mathrm{ZnO} \mathrm{NP}$ exposure induced-monoamine disruption with $\mathrm{ROS}$ and $\mathrm{Zn}^{2+}$ will clarify these issues.

In conclusion, the present data indicated that prenatal exposure to ZnO NPs disrupted the levels of monoamine neurotransmitters of the brain and suggested that $\mathrm{ZnO}$ NPs could have potential toxicity with regard to mental health. Taken together with the report that ZnO NPs show higher developmental toxicity than other metal oxide NPs including $\mathrm{TiO}_{2}$ and $\mathrm{Al}_{2} \mathrm{O}_{3}$ (Zhu et al., 2008), the assumption would follow that avoidance of toxicity induced by $\mathrm{ZnO}$ NPs is an important for the issue in mental health. The toxicity of NPs is related to its characteristics of deposition and translocation to the tissues (Oberdörster et al., 2005). We previously reported that subcutaneously injected $\mathrm{TiO}_{2}$ NPs were translocated to the brain of offspring (Takeda et al., 2009). ZnO NPs may also be transferred to the brain of offspring and affect the monoaminergic systems. Recent studies indicated that the interaction of NP surface with the proteins is important for the distribu- tion of NPs in the body. Several reports showed that surface coating of NPs changed its accumulation and translocation to the tissues and its toxicity (Oberdörster et al., 2009; Choi et al., 2010). In order for ZnO NPs to be used safely, further studies are needed to clarify the molecular mechanisms of monoamine neurotransmitter disruption and to determine how the toxicity of $\mathrm{ZnO}$ NPs might be reduced.

\section{ACKNOWLEDGMENTS}

We thank to Prof. Hirofumi Yajima for help with analysis of particle size distribution. We also thank Drs. Kenichiro Suzuki and Miyoko Kubo-Irie for analysis of $\mathrm{ZnO}$ particles and valuable discussion, and Yuta Takahashi and Takanori Shinotsuka for technical assistance. The authors appreciate the graduate and undergraduate students in the Takeda laboratory for their help with the experiments. This work was supported in part by a Grant-in-Aid for Science Research from the Ministry of Education, Culture, Sports, Science and Technology of Japan, a Grant-in-Aid for the Private University Science Research Upgrade Promotion Business Academic Frontier Project, a MEXT-Supported Program for the Strategic Research Foundation at Private Universities, 2011-2015, and a Grant-in-aid for the Health and Labour Sciences Research Grant, Research on the Risk of Chemical Substances, from the Ministry of Health, Labour and Welfare.

\section{REFERENCES}

Assié, M.B., Ravailhe, V., Faucillon, V. and Newman-Tancredi, A. (2005): Contrasting contribution of 5-hydroxytryptamine 1 A receptor activation to neurochemical profile of novel antipsychotics: frontocortical dopamine and hippocampal serotonin release in rat brain. J. Pharmacol. Exp. Ther., 315, 265-272.

Barton, D.A., Esler, M.D., Dawood, T., Lambert, E.A., Haikerwal, D., Brenchley, C., Socratous, F., Hastings, J., Guo, L., Wiesner, G., Kaye, D.M., Bayles, R., Schlaich, M.P. and Lambert, G.W. (2008): Elevated brain serotonin turnover in patients with depression: effect of genotype and therapy. Arch. Gen. Psychiatry, 65, 38-46.

Beckett, W.S., Chalupa, D.F., Pauly-Brown, A., Speers, D.M., Stewart, J.C., Frampton, M.W., Utell, M.J., Huang, L.S., Cox, C., Zareba, W. and Oberdörster, G. (2005): Comparing inhaled ultrafine versus fine zinc oxide particles in healthy adults: a human inhalation study. Am. J. Respir. Crit. Care Med., 171, 1129-1135.

Cho, W.S., Duffin, R., Howie, S.E., Scotton, C.J., Wallace, W.A., Macnee, W., Bradley, M., Megson, I.L. and Donaldson, K. (2011): Progressive severe lung injury by zinc oxide nanoparticles; the role of $\mathrm{Zn}^{2+}$ dissolution inside lysosomes. Part. Fibre Toxicol., 8, 27.

Choi, H.S., Ashitate, Y., Lee, J.H., Kim, S.H., Matsui, A., Insin, 
Prenatal $\mathrm{ZnO}$ nanoparticle exposure alters monoamines in mouse brain

N., Bawendi, M.G., Semmler-Behnke, M., Frangioni, J.V. and Tsuda, A. (2010): Rapid translocation of nanoparticles from the lung airspaces to the body. Nat. Biotechnol., 28, 1300-1303.

Chung, S., Son, G.H., Park, S.H., Park, E., Lee, K.H., Geum, D. and Kim, K. (2005): Differential adaptive responses to chronic stress of maternally stressed male mice offspring. Endocrinology, 146, 3202-3210.

Dazzi, L., Seu, E., Cherchi, G., Barbieri, P.P., Matzeu, A. and Biggio, G. (2007): Estrous cycle-dependent changes in basal and ethanol-induced activity of cortical dopaminergic neurons in the rat. Neuropsychopharmacology, 32, 892-901.

De La Garza, R., 2nd and Asnis, G.M. (2003): The non-steroidal anti-inflammatory drug diclofenac sodium attenuates IFN-alpha induced alterations to monoamine turnover in prefrontal cortex and hippocampus. Brain Res., 977, 70-79.

Elsworth, J.D. and Roth, R.H. (1997): Dopamine synthesis, uptake, metabolism, and receptors: relevance to gene therapy of Parkinson's disease. Exp. Neurol., 144, 4-9.

Froehlich, T.E., McGough, J.J. and Stein, M.A. (2010): Progress and promise of attention-deficit hyperactivity disorder pharmacogenetics. CNS Drugs, 24, 99-117.

Hosie, A.M., Dunne, E.L., Harvey, R.J. and Smart, T.G. (2003): Zinc-mediated inhibition of $\mathrm{GABA}_{\mathrm{A}}$ receptors: discrete binding sites underlie subtype specificity. Nat. Neurosci., 6, 362-369.

Kawashiro, Y., Fukata, H., Omori-Inoue, M., Kubonoya, K., Jotaki, T., Takigami, H., Sakai, S. and Mori, C. (2008): Perinatal exposure to brominated flame retardants and polychlorinated biphenyls in Japan. Endocr. J., 55, 1071-1084.

Kreyling, W.G., Semmler-Behnke, M., Seitz, J., Scymczak, W., Wenk, A., Mayer, P., Takenaka, S. and Oberdörster, G. (2009): Size dependence of the translocation of inhaled iridium and carbon nanoparticle aggregates from the lung of rats to the blood and secondary target organs. Inhal. Toxicol., 21 Suppl. 1, 55-60.

Kuan, C.Y., Yee-Fung, W., Yuen, K.H. and Liong, M.T. (2012): Nanotech: propensity in foods and bioactives. Crit. Rev. Food Sci. Nutr., 52, 55-71.

Liu, H., Ma, L., Zhao, J., Liu, J., Yan, J., Ruan, J. and Hong, F. (2009): Biochemical toxicity of nano-anatase $\mathrm{TiO}_{2}$ particles in mice. Biol. Trace Elem. Res., 129, 170-180.

Long, T.C., Saleh, N., Tilton, R.D., Lowry, G.V. and Veronesi, B. (2006): Titanium dioxide (P25) produces reactive oxygen species in immortalized brain microglia (BV2): implications for nanoparticle neurotoxicity. Environ. Sci. Technol., 40, 4346-4352.

Matsuda, S., Saika, S., Amano, K., Shimizu, E. and Sajiki, J. (2010): Changes in brain monoamine levels in neonatal rats exposed to bisphenol A at low doses. Chemosphere, 78, 894-906.

Morabito, K., Shapley, N.C., Steeley, K.G. and Tripathi, A. (2011): Review of sunscreen and the emergence of non-conventional absorbers and their applications in ultraviolet protection. Int. J. Cosmet. Sci., 33, 385-390.

Morilak, D.A. and Frazer, A. (2004): Antidepressants and brain monoaminergic systems: a dimensional approach to understanding their behavioural effects in depression and anxiety disorders. Int. J. Neuropsychopharmacol., 7, 193-218.

Nakamura, K., Sekine, Y., Ouchi, Y., Tsujii, M., Yoshikawa, E., Futatsubashi, M., Tsuchiya, K.J., Sugihara, G., Iwata, Y., Suzuki, K., Matsuzaki, H., Suda, S., Sugiyama, T., Takei, N. and Mori, N. (2010): Brain serotonin and dopamine transporter bindings in adults with high-functioning autism. Arch. Gen. Psychiatry, 67, 59-68.

Narita, N., Kato, M., Tazoe, M., Miyazaki, K., Narita, M. and Okado, N. (2002): Increased monoamine concentration in the brain and blood of fetal thalidomide- and valproic acid-exposed rat: putative animal models for autism. Pediatr. Res., 52, 576579.

Needham, L.L. and Sexton, K. (2000): Assessing children's exposure to hazardous environmental chemicals: an overview of selected research challenges and complexities. J. Expo. Anal. Environ. Epidemiol., 10, 611-629.

Nohynek, G.J., Lademann, J., Ribaud, C. and Roberts, M.S. (2007): Grey goo on the skin? Nanotechnology, cosmetic and sunscreen safety. Crit. Rev. Toxicol., 37, 251-277.

Oades, R.D. (2008): Dopamine-serotonin interactions in attentiondeficit hyperactivity disorder (ADHD). Prog. Brain Res., 172, 543-565.

Oberdörster, G. (2001): Pulmonary effects of inhaled ultrafine particles. Int. Arch. Occup. Environ. Health, 74, 1-8.

Oberdörster, G., Elder, A. and Rinderknecht, A. (2009): Nanoparticles and the brain: cause for concern? J. Nanosci. Nanotechnol., 9, 4996-5007.

Oberdörster, G., Oberdörster, E. and Oberdörster, J. (2005): Nanotoxicology: an emerging discipline evolving from studies of ultrafine particles. Environ. Health Perspect., 113, 823-839.

Paclt, I., Koudelova, J., Pacltova, D. and Kopeckova, M. (2009): Dopamine beta hydroxylase (DBH) plasma activity in childhood mental disorders. Neuro Endocrinol. Lett., 30, 604-609.

Santiago, M., Machado, A. and Cano, J. (1993): Regulation of the prefrontal cortical dopamine release by $\mathrm{GABA}_{\mathrm{A}}$ and $\mathrm{GABA}_{\mathrm{B}}$ receptor agonists and antagonists. Brain Res., 630, 28-31.

Scholze, P., Norregaard, L., Singer, E.A., Freissmuth, M., Gether, U. and Sitte, H.H. (2002): The role of zinc ions in reverse transport mediated by monoamine transporters. J. Biol. Chem., 277, 21505-21513.

Shimizu, M., Tainaka, H., Oba, T., Mizuo, K., Umezawa, M. and Takeda, K. (2009): Maternal exposure to nanoparticulate titanium dioxide during the prenatal period alters gene expression related to brain development in the mouse. Part. Fibre Toxicol., 6, 20 .

Sirivelu, M.P., MohanKumar, S.M., Wagner, J.G., Harkema, J.R. and MohanKumar, P.S. (2006): Activation of the stress axis and neurochemical alterations in specific brain areas by concentrated ambient particle exposure with concomitant allergic airway disease. Environ. Health Perspect., 114, 870-874.

Son, G.H., Chung, S., Geum, D., Kang, S.S., Choi, W.S., Kim, K. and Choi, S. (2007): Hyperactivity and alteration of the midbrain dopaminergic system in maternally stressed male mice offspring. Biochem. Biophys. Res. Commun., 352, 823-829.

Son, G.H., Geum, D., Chung, S., Kim, E.J., Jo, J.H., Kim, C.M., Lee, K.H., Kim, H., Choi, S., Kim, H.T., Lee, C.J. and Kim, K. (2006): Maternal stress produces learning deficits associated with impairment of NMDA receptor-mediated synaptic plasticity. J. Neurosci., 26, 3309-3318.

Takahashi, Y., Mizuo, K., Shinkai, Y., Oshio, S. and Takeda, K. (2010): Prenatal exposure to titanium dioxide nanoparticles increases dopamine levels in the prefrontal cortex and neostriatum of mice. J. Toxicol. Sci., 35, 749-756.

Takeda, K., Suzuki, K.I., Ishihara, A., Kubo-Irie, M., Fujimoto, R., Tabata, M., Oshio, S., Nihei, Y., Ihara, T. and Sugamata, M. (2009): Nanoparticles transferred from pregnant mice to their offspring can damage the genital and cranial nerve systems. J. Health Sci., 55, 95-102.

Tin Tin Win, S., Mitsushima, D., Yamamoto, S., Fukushima, A., Funabashi, T., Kobayashi, T. and Fujimaki, H. (2008): Changes in neurotransmitter levels and proinflammatory cytokine mRNA 
Y. Okada et al.

expressions in the mice olfactory bulb following nanoparticle exposure. Toxicol. Appl. Pharmacol., 226, 192-198.

Tin Tin Win, S., Yamamoto, S., Ahmed, S., Kakeyama, M., Kobayashi, T. and Fujimaki, H. (2006): Brain cytokine and chemokine mRNA expression in mice induced by intranasal instillation with ultrafine carbon black. Toxicol. Lett., 163, 153160.

Weiss, J.M., Goodman, P.A., Losito, B.G., Corrigan, S., Charry, J.M. and Bailey, W.H. (1981): Behavioral depression produced by an uncontrollable stressor: relationship to norepinephrine, dopamine, and serotonin levels in various regions of rat brain. Brain Res. Rev., 3, 167-205.

Xia, T., Kovochich, M., Liong, M., Madler, L., Gilbert, B., Shi, H., Yeh, J.I., Zink, J.I. and Nel, A.E. (2008): Comparison of the mechanism of toxicity of zinc oxide and cerium oxide nanoparticles based on dissolution and oxidative stress properties. ACS Nano, 2, 2121-2134.

Xia, Y., Cheng, S., He, J., Liu, X., Tang, Y., Yuan, H., He, L., Lu,
T., Tu, B. and Wang, Y. (2011): Effects of subchronic exposure to benzo[a]pyrene $(\mathrm{B}[\mathrm{a}] \mathrm{P})$ on learning and memory, and neurotransmitters in male Sprague-Dawley rat. Neurotoxicology, 32, 188-198.

Xiao, L. and Becker, J.B. (1994): Quantitative microdialysis determination of extracellular striatal dopamine concentration in male and female rats: effects of estrous cycle and gonadectomy. Neurosci. Lett., 180, 155-158.

Yang, H., Liu, C., Yang, D., Zhang, H. and Xi, Z. (2009): Comparative study of cytotoxicity, oxidative stress and genotoxicity induced by four typical nanomaterials: the role of particle size, shape and composition. J. Appl. Toxicol., 29, 69-78.

Zhu, X., Zhu, L., Duan, Z., Qi, R., Li, Y. and Lang, Y. (2008): Comparative toxicity of several metal oxide nanoparticle aqueous suspensions to Zebrafish (Danio rerio) early developmental stage. J. Environ. Sci. Health A Tox. Hazard Subst. Environ. Eng., 43, 278-284. 